\title{
Benefit of Risk Score-Guided Prophylaxis in Pregnant Women at Risk of Thrombotic Events: A Controlled Before-and-After Implementation Study
}

\author{
Céline Chauleur ${ }^{1,2,3}$ Jean-Christophe Gris ${ }^{4,5}$ Silvy Laporte ${ }^{1,2,6} \quad$ Céline Chapelle $^{6}$ \\ Laurent Bertoletti $^{1,6}$ Véronique Equy ${ }^{7}$ Pascal Gaucherand $^{8}$ Eva Bazan ${ }^{9}$ Olivier Dupuis ${ }^{10}$ \\ Denis Gallot ${ }^{11}$ Patrick Mismetti ${ }^{1,2}$ The STRATHEGE Investigators and The STRATHEGE Group
}

1 INSERM U1059, Saint-Etienne, France

2 University of Lyon, Saint-Etienne, France

${ }^{3}$ Department of Gynecology and Obstetrics, University Hospital of Saint-Etienne, Saint-Etienne, France

${ }^{4}$ Department of Hematology, University Hospital of Nîmes, Nîmes, France

${ }^{5}$ UPRES EA2990, University of Montpellier, Montpellier, France

${ }^{6}$ Clinical Pharmacology Department, University Hospital of Saint-

Etienne, Saint-Etienne, France

${ }^{7}$ Department of Gynecology and Obstetrics, Grenoble University

Hospital, Grenoble, France

8 Department of Gynecology and Obstetrics, Hospices Civils de Lyon, Hôpital Femme Mère Enfant, Bron Cedex, France

${ }^{9}$ Department of Gynecology and Obstetrics, Montélimar Hospital, Montélimar, France

${ }^{10}$ Department of Gynecology and Obstetrics, Hospices Civils de Lyon, Hôpital Lyon-Sud, Pierre-Bénite, France

11 Department of Gynecology and Obstetrics, University Hospital of Clermont-Ferrand, Clermont-Ferrand, France
Address for correspondence Céline Chauleur, MD, PhD, Department of Gynecology and Obstetrics, Saint-Etienne University Hospital Centre, North Hospital, Avenue Albert Raimond, 42270 Saint Priest en Jarez, France (e-mail: celine.chauleur@chu-st-etienne.fr).

Thromb Haemost 2018;118:1564-1571.

\begin{abstract}
Keywords

- guidelines

- pregnancy

- risk assessment

- risk score

- venous thromboembolism

Background Management of pregnant women at risk of venous thromboembolism (VTE) and placental vascular complications (PVCs) remains complex. Guidelines do not definitively specify optimal strategies.

Objective Our objective was to evaluate the impact of employing risk score-driven prophylaxis strategies on VTE and PVC rates in at-risk pregnant women.

Materials and Methods This study, conducted in 21 French maternity units, compared VTE and PVC rates before and after implementation of a risk scoring system to determine prophylactic strategies.

Results A total of 2,085 pregnant women at risk of VTE or PVC were enrolled. Vascular events occurred in 190 (19.2\%) patients before and 140 (13.0\%) after implementation of risk score-driven prophylaxis (relative risk $[R R]=0.68[0.55 ; 0.83]$ ). The incidence of deep vein thrombosis during pregnancy was reduced (RR $=0.30[0.14 ; 0.67])$. PVC comprised mainly pre-eclampsia, occurring in 79 patients before and 42 patients after risk score implementation $(\mathrm{RR}=0.52$ [0.36; 0.75]). Post-partum haemorrhage occurred in 32 patients $(3.2 \%)$ before and 48 patients (4.5\%) after risk score implementation ( $R R=1.38[0.89 ; 2.13], p=0.15)$. Conclusion Use of a simple risk scoring system, developed by experts in VTE and PVC research to guide prophylaxis, reduced the risk of thrombotic events during pregnancy without any significant increase in bleeding risk.
\end{abstract}

received

November 23, 2017

accepted after revision

July 5, 2018 (c) 2018 Georg Thieme Verlag KG Stuttgart - New York
DOI https://doi.org/

10.1055/s-0038-1668524. ISSN 0340-6245. 


\section{Introduction}

Pregnancy significantly increases the risk of venous thromboembolism (VTE) and may be accompanied by placental vascular complications (PVCs), such as pre-eclampsia, foetal growth restriction (intrauterine growth restriction [IUGR]), placental abruption, intrauterine foetal death (IUFD) and pregnancy loss. Various national or international recommendations concerning VTE prophylaxis during pregnancy are available $^{1-4}$. However, their application is not always straightforward, particularly in patients cumulating several identified risk factors for VTE and PVC, and the optimal strategy for complex clinical cases remains unclear. Furthermore, their complexity may discourage their routine use by primary care practitioners and gynaecologists less familiar with VTE. Systematic risk assessment has been recommended since $2008,{ }^{5}$ and several risk-scoring systems have been published. ${ }^{6-12}$ However, the clinical value of these scores is questionable due to their lack of validation and compliance with international guidelines.

Using a Delphi approach, we developed an easy-to-use tool, the STRATHEGE score, enabling individualized estimation of thrombotic risk during pregnancy and permitting implementation of a risk-adapted strategy for anti-thrombotic prophylaxis during pregnancy and puerperium. ${ }^{12}$ This score includes four sub-groups of items: personal and family history of thrombotic events; acquired or inherited thrombophilia; personal history of PVCs; and other clinical risk factors (see - Appendix A). It was constructed by an expert panel of clinicians and biologists involved in both VTE and pregnancy management, respecting the most recent international guidelines in this area.

As the clinical benefit of this STRATHEGE score-guided prophylaxis remained to be prospectively demonstrated, ${ }^{6}$ we conducted a before-and-after implementation clinical trial to show its effectiveness in terms of the rate of thrombotic and vascular events in at-risk pregnant women (Clinicaltrials.gov registry no. NCT00745212).

\section{Materials and Methods}

\section{Study Design}

This French multi-centre before-and-after implementation study was performed in maternity units corresponding to levels I to IV obstetric care and comprised three successive stages: before implementation of the scoring system (BEFORE stage), implementation of this system and after its implementation (AFTER stage). In the BEFORE stage, eligible adult women were consecutively included 24 hours after admission to the delivery unit, thromboprophylactic regimens during puerperium being prescribed at the physician's discretion. In principle, all relevant data required for calculation of the score were recorded at the time of delivery, as were data on the treatment received. However, as the scoring system was voluntarily finalized only at the end of the BEFORE phase so as not to influence practices in that phase, certain additional data had to be extracted retrospectively from medical records to complete the score (i.e. family history of VTE). Symptomatic events occurring during pregnancy and the 6-week follow-up after delivery were recorded.

In the implementation stage, all midwives and obstetricians in the maternity units participating in the study were trained in use of the risk scoring system, designed to identify women at risk of VTE and PVC and lead to a specific proposal for prophylaxis based on the overall score (see -Appendix A). This therapeutic approach comprised (1) identification of at-risk women using a simple questionnaire, (2) addition of individual scores for identified risk factors in the following four categories: personal or family history of VTE, presence of thrombophilia, history of PVC and presence of other clinical risk factors and (3) use of our scoring system to determine the optimal prophylactic regimen for each patient. Pocket-sized cards presenting the scoring system were provided. This stage also included seminars on VTE and PVC prophylaxis according to the Eighth American College of Chest Physicians (ACCP) recommendations. ${ }^{5}$

In the AFTER stage, eligible adult women were consecutively included during any pregnancy-related medical consultation and practitioners were asked to prescribe the thromboprophylactic regimens suggested by the scoring system.

\section{Patients}

Adult women with at least one risk factor for VTE or PVC were eligible for inclusion. According to the STRATHEGE scoring system, ${ }^{6}$ risk factors included (1) personal history of VTE and family history of VTE; (2) acquired or inherited thrombophilia including anti-thrombin deficiency, protein C or S deficiency, heterozygous or homozygous factor $\mathrm{V}$ Leiden or pro-thrombin 20210A polymorphism, lupus anticoagulant, anti-cardiolipin antibodies, anti- 32 -glycoprotein I antibodies, and hyperhomocysteinaemia; (3) history of PVC, defined as vascular IUFD or repeated miscarriages $>2$, pre-eclampsia, HELLP (haemolysis, elevated liver enzymes, low platelet count) syndrome, placental abruption and IUGR (estimated foetal weight $<10$ th percentile); and (4) high body mass index ( $>30$ ) or age above 35 years and two other minor clinical risk factors. Patients were informed of the study design and gave their oral consent to participate.

Exclusion criteria were unknown personal or familial history, non-vascular IUFD, presence of mechanical heart valves requiring anticoagulation, any contraindication for anticoagulant administration, such as known allergy to heparin, low-molecular-weight heparin (LMWH) or aspirin and impossibility of follow-up.

\section{STRATHEGE Score}

The overall risk score for VTE and PVC was determined for each woman enrolled in the BEFORE or AFTER stage and rates of concordance between the prophylactic regimens indicated by overall risk scores and those actually implemented during pregnancy and puerperium were recorded.

\section{Endpoints}

The primary endpoint was a composite endpoint including the occurrence of symptomatic VTE and/or PVC during pregnancy or puerperium (defined as six completed weeks after delivery). 
Symptomatic VTE was defined as symptomatic pulmonary embolism (PE), deep or superficial vein thrombosis (DVT or SVT) and cerebral venous thrombosis objectively confirmed by ultrasonography (DVT and SVT), or positive ventilation/perfusion lung scan (PE), or computed tomography scan or lowerlimb venous ultrasonography (DVT or SVT), or magnetic resonance imaging (cerebral venous thrombosis).

PVCs were defined as three or more consecutive pregnancy losses between 5 and 12 weeks of amenorrhea (WA), one or more vascular IUFD after 12 WA (i.e. IUFD not explained by malformation, chromosomal abnormality or infectious disease), pre-eclampsia, placental abruption, HELLP syndrome or IUGR. Pre-eclampsia was defined as new-onset diastolic blood pressure of at least $90 \mathrm{~mm} \mathrm{Hg}$ after 20 weeks of gestation, controlled on two occasions at least 4 hours apart, accompanied by significant proteinuria ( $>0.3 \mathrm{~g}$ in a 24-hour urine sample) or new-onset proteinuria in women with pre-existing hypertension. Pre-eclampsia was defined as severe when associated with severe hypertension (diastolic $>110 \mathrm{~mm} \mathrm{Hg}$ or systolic $>160$ $\mathrm{mm} \mathrm{Hg}$ ), eclampsia (seizures), pulmonary oedema, proteinuria $\geq 5$ g per 24 hours, renal insufficiency based on abnormal blood creatinine levels, abnormally high liver enzyme levels (aspartate aminotransferase [ASAT] or alanine aminotransferase [ALAT] $\geq 70 \mathrm{IU} / \mathrm{L})$ with abdominal pain, low platelet counts $(<100 \mathrm{G} / \mathrm{L})$ and/or delivery at $<34$ weeks of gestation. Placental abruption was diagnosed on detection of a circumscribed depression on the maternal surface of the freshly delivered placenta, covered by dark clotted blood; when placental abruption was very recent, signs and symptoms recorded before delivery contributed to the diagnosis, particularly the association of vaginal bleeding and uterine tenderness or back pain, or foetal distress or death, or uterine hypertonus. HELLP syndrome was defined by the presence of haemolysis (characteristic peripheral blood smear and serum lactate dehydrogenase $\geq 600 \mathrm{U} / \mathrm{L}$ or serum total bilirubin $\geq 1.2 \mathrm{mg} / \mathrm{dL}$ ), elevated liver enzymes (serum ASAT or ALAT $\geq 70 \mathrm{U} / \mathrm{L})$ and low platelet counts $(<100,000$ cells $/ \mu \mathrm{L}$ ). IUGR was defined as birth weight $\leq 10$ th percentile according to AUDIPOG charts, taking into account maternal age, neonatal gender and gestational age at delivery.

Secondary endpoints were post-partum haemorrhage and treatment-related complications. Post-partum haemorrhage was defined as blood loss above $500 \mathrm{~mL}$, necessitating examination of the uterus, with any of the following: peri-partum haemoglobin drop of more than $20 \mathrm{~g} / \mathrm{L}$, transfusion, embolization, conservative surgical procedure, hysterectomy or death. Treatment-related complications were defined as life-threatening bleeding or bleeding requiring medical intervention, heparin-induced thrombocytopenia (defined according to the ACCP guidelines), skin complications, heparin or aspirin allergy, congenital anomalies or birth defects.

All events were validated by an independent central adjudication committee, unaware of anticoagulant or antiplatelet treatment.

To avoid overlooking recorded events in the BEFORE stage, medical records and codes from the International Classification of Diseases, 10th Revision, Clinical Modification: 000-099: (003; 008; 012-16; 0022; 0087) were reviewed for patients included in this stage.

\section{Statistical Analysis}

Based on a previous study, indicating a 5.6\% incidence of VTE events among at-risk women, ${ }^{12}$ we assumed at least a $5 \%$ rate of VTE events plus PVC in our population before implementation of our risk score. Assuming a 50\% reduction in the primary endpoint with risk score-driven thromboprophylaxis, and a two-sided type I error of 5\%, we calculated that a sample size of 900 patients per stage would have a $90 \%$ power to detect a difference between the combined VTE and PVC rates observed before and after implementation of the score. We therefore planned to recruit 2,000 patients in total to obtain 1,800 evaluable patients.

The composite primary endpoint (occurrence of VTE or PVC), as well as the other endpoints, were compared before and after implementation of the score using the chi-square test; absolute differences and relative risks (RRs) with their 95\% confidence intervals (CIs) were calculated. The number needed to treat (NNT) associated with the use of the score was also estimated. To describe the concordance between the prophylactic treatment indicated by the overall risk score and that actually prescribed, the Kappa coefficient (with its 95\% CI) was calculated. A Kappa coefficient close to 1 indicated (almost) perfect agreement, while a coefficient close to 0 indicating disagreement.

Statistical analyses were performed using the SAS software, version 9.4 (SAS Institute, Cary, North Carolina, United States).

\section{Ethics}

The study was approved by the Saint Etienne University Hospital Centre institutional review board and ethics committee and by the local committee for the protection of clinical trial participants (Comité de Protection des Personnes). It complied with the 2013 Helsinki Declaration (No. 08/120).

\section{Results}

From May 2008 to March 2013, 989 pregnant women at risk of VTE or PVC were enrolled in the BEFORE stage of the trial, prior to implementation of the risk scoring system, and 1,096 in the AFTER stage, following implementation of this system (-Fig. 1). The study was conducted in 21 French maternity units, predominantly corresponding to type III obstetric care (BEFORE stage: $62.4 \%$, AFTER stage: $67.3 \%$; - Table 1). The mean gestational period at delivery was 37.4 and 37.7 weeks, respectively. To allow comparison of the patients included in the two stages, we calculated a posteriori the score for the BEFORE stage population. A risk score of 0 (corresponding to a recommendation for no heparin treatment) was calculated for $51.3 \%$ of women in the BEFORE stage and $45.3 \%$ in the AFTER stage. According to the calculated risk score, 26.0 and $23.7 \%$ of patients, respectively, required minimal prophylaxis (prophylactic treatment during the 6-week puerperium; -Table 2).

\section{Outcomes}

\section{Primary Endpoint}

The primary endpoint was evaluable in all 989 patients BEFORE implementation of the score and in 1,078/1,089 patients AFTER 


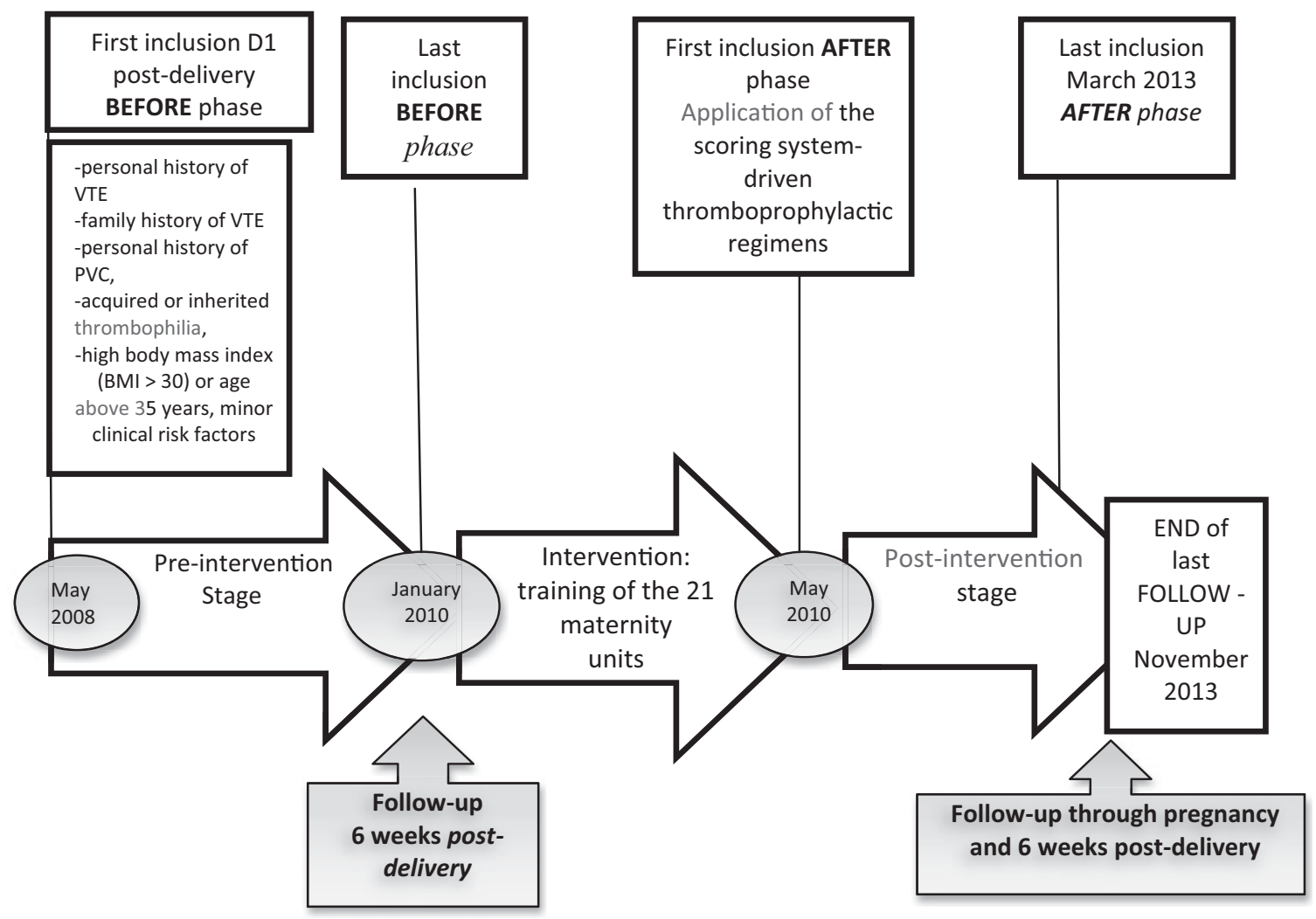

Fig. 1 Study design and calendar.

Table 1 Site and patient characteristics

\begin{tabular}{|c|c|c|}
\hline & $\begin{array}{l}\text { Before } \\
\text { intervention } \\
(N=989)\end{array}$ & $\begin{array}{l}\text { After } \\
\text { intervention } \\
(N=1,089)\end{array}$ \\
\hline \multicolumn{3}{|l|}{ Maternity type, \% } \\
\hline I & 11.9 & 12.6 \\
\hline II & 25.7 & 20.1 \\
\hline III & 62.4 & 67.3 \\
\hline Age $(y)$, mean $\pm S D$ & $32.0 \pm 5.5$ & $31.3 \pm 5.1$ \\
\hline \multicolumn{3}{|c|}{ Total no. of pregnancies, $\%$} \\
\hline 1 & 17.5 & 21.2 \\
\hline 2 & 25.3 & 29.9 \\
\hline 3 & 22.5 & 20.7 \\
\hline 4 & 13.7 & 11.9 \\
\hline$\geq 5$ & 21.0 & 16.3 \\
\hline \multicolumn{3}{|l|}{ Parity, \% } \\
\hline 0 & 25.8 & 28.0 \\
\hline 1 & 41.7 & 40.0 \\
\hline 2 & 21.3 & 19.7 \\
\hline 3 & 6.3 & 8.7 \\
\hline$\geq 4$ & 5.0 & 3.6 \\
\hline $\begin{array}{l}\text { Delivery term } \\
\text { (wk of gestation), } \\
\text { mean } \pm S D\end{array}$ & $37.4 \pm 3.7$ & $37.7 \pm 4.3$ \\
\hline
\end{tabular}

Abbreviation: SD, standard deviation.
Table 2 STRATHEGE risk scoring system and prophylaxis suggested by the overall score

\begin{tabular}{|l|l|l|}
\hline & $\begin{array}{l}\text { Before } \\
\text { intervention } \\
(N=989)\end{array}$ & $\begin{array}{l}\text { After } \\
\text { intervention } \\
(N=1,089)\end{array}$ \\
\hline $\begin{array}{l}\text { Anti-thrombotic prophylaxis } \\
\text { suggested by the overall } \\
\text { risk score, \% }\end{array}$ & $\begin{array}{l}\text { Applied } \\
\text { a posteriori }\end{array}$ & After \\
\hline 0, No heparin treatment & 51.3 & 45.3 \\
\hline $\begin{array}{l}\text { 1-3, Prophylactic heparin } \\
\text { during PP (6 wk) }\end{array}$ & 26.0 & 23.7 \\
\hline $\begin{array}{l}\text { 4, Prophylactic heparin } \\
\text { during 3rd trimester + PP } \\
\text { (6 wk) }\end{array}$ & 2.9 & 2.7 \\
\hline $\begin{array}{l}\text { 5-11, Prophylactic heparin } \\
\text { throughout pregnancy + PP }\end{array}$ & 15.9 & 22.9 \\
\hline $\begin{array}{l}\geq 12, \text { Weight-adjusted } \\
\text { heparin prophylaxis during } \\
\text { pregnancy + PP }\end{array}$ & 3.9 & 5.5 \\
\hline Aspirin, \% & 13.0 & 42.9 \\
\hline
\end{tabular}

Abbreviations: PP, puerperium; SD, standard deviation. 
this (10 patients were lost to follow-up for the post-partum period, and for one patient, the hospital record for one visit was not found). The composite primary endpoint of VTE or PVC occurred in 190 (19.2\%) patients in the BEFORE stage and 140 (13.0\%) patients in the AFTER stage ( - Table 3 ), yielding a RR reduction of $32 \%(\mathrm{RR}=0.68$ [95\% $\mathrm{Cl}, 0.55 ; 0.83], p=0.0001)$. The NNT associated with the use of the score was 16 , meaning that using the score in 16 patients would avoid the occurrence of one VTE or PVC. VTE was diagnosed during pregnancy/ puerperium in 37 patients (3.7\%) BEFORE and 19 patients (1.8\%) AFTER implementation of the scoring system. The corresponding RR was 0.47 [0.27; 0.81$], p=0.006$. The NNT was 51. For VTE, the effect of implementing this scoring system was mainly observed during pregnancy, the rate of VTE then being $2.7 \%$ for BEFORE and $0.9 \%$ for AFTER $(R R=0.34[0.16$; 0.69 ], $p=0.002$; - Table 3). PVC occurred in $16.3 \%$ of patients BEFORE and $11.5 \%$ of patients AFTER implementation of the scoring system $(\mathrm{RR}=0.71$ [0.57; 0.88], $p=0.002)$. The NNT was 21 , indicating that use of the score in 21 patients would avoid the occurrence of one PVC. PVC mainly comprised IUGR with or without pre-eclampsia.

\section{Secondary Endpoints}

Overall, 80 patients experienced post-partum haemorrhage, without any statistical difference between the BEFORE (32 patients, 3.2\%) and AFTER (48 patients, 4.5\%) periods (RR $=1.38$ [0.89; 2.13], $p=0.15$; - Table 3). Twelve of these patients had received LMWH during pregnancy, but discontinued this treatment for delivery and epidural analgesia. The other 68 women experienced post-partum haemorrhage without having received any pharmacological prophylaxis before delivery. Five patients in the BEFORE stage and 3 patients in the AFTER period presented another treatmentrelated complication (-Table 3 ). No patient died in either period.

\section{Anticoagulant and Anti-Platelet Prophylaxis}

During pregnancy and puerperium, 584 patients (59.0\%) in the BEFORE period and 766 (71.1\%) in the AFTER period received anticoagulant prophylaxis. In the AFTER period, compliance with the anticoagulant treatment suggested by the scoring system was $73.1 \%$ overall and $42.1 \%$ for patients presenting VTE. Similarly, 283 (28.6\%) and 491 patients

Table 3 Endpoints

\begin{tabular}{|c|c|c|c|c|c|}
\hline & $\begin{array}{l}\text { Before } \\
\text { intervention } \\
(N=989)\end{array}$ & $\begin{array}{l}\text { After } \\
\text { intervention } \\
(N=1,089)\end{array}$ & $\begin{array}{l}\text { Absolute } \\
\text { difference } \\
(95 \% \mathrm{Cl})\end{array}$ & RR (95\% Cl) & $p$-Value \\
\hline \multicolumn{6}{|l|}{ Composite primary endpoint } \\
\hline At least one VTE or PVC & $190(19.2 \%)$ & $140(13.0 \%)$ & $-6.2 \%(-9.4 ;-3.1)$ & $0.68(0.55 ; 0.83)$ & 0.0001 \\
\hline VTE & $37(3.7 \%)$ & $19(1.8 \%)$ & $-1.9 \%(-3.4 ;-0.6)$ & $0.47(0.27 ; 0.81)$ & 0.006 \\
\hline DVT & 16 & 9 & & & \\
\hline SVT only & 16 & 5 & & & \\
\hline $\mathrm{PE}$ & 5 & 4 & & & \\
\hline Cerebral venous thrombosis & 0 & 1 & & & \\
\hline VTE during pregnancy & $27(2.7 \%)$ & $10(0.9 \%)$ & $-1.8 \%(-3.0 ;-0.6)$ & $0.34(0.16 ; 0.69)$ & 0.002 \\
\hline VTE during puerperium & $10(1.0 \%)$ & $9(0.8 \%)$ & $-0.2 \%(-1.0 ; 0.7)$ & $0.83(0.34 ; 2.02)$ & 0.67 \\
\hline PVC & $161(16.3 \%)$ & $124(11.5 \%)$ & $-4.8 \%(-7.8 ;-1.8)$ & $0.71(0.57 ; 0.88)$ & 0.002 \\
\hline Intrauterine growth restriction & 86 & 76 & & & \\
\hline Pre-eclampsia & 79 & 42 & & & \\
\hline$<34$ wk of gestation & 46 & 29 & & & \\
\hline Intrauterine foetal death & 11 & 11 & & & \\
\hline Placental abruption & 8 & 4 & & & \\
\hline Spontaneous miscarriage & 0 & 9 & & & \\
\hline \multicolumn{6}{|l|}{ Secondary endpoints } \\
\hline Post-partum haemorrhage & $32(3.2 \%)$ & $48(4.5 \%)$ & $1.2 \%(-0.4 ; 2.9)$ & $1.38(0.89 ; 2.13)$ & 0.15 \\
\hline Treatment-related complications & $5(0.5 \%)$ & $3(0.3 \%)$ & $-0.2 \%(-0.8 ; 0.3)$ & $0.55(0.13 ; 2.30)$ & 0.41 \\
\hline Bleeding & 1 & 0 & & & \\
\hline HIT & 1 & 0 & & & \\
\hline LMWH or UFH allergy & 3 & 2 & & & \\
\hline Minor bleeding (haemorrhoids) & 0 & 1 & & & \\
\hline
\end{tabular}

Abbreviations: $\mathrm{Cl}$, confidence interval; DVT, deep vein thrombosis; HIT, heparin-induced thrombocytopenia; LMWH, low-molecular-weight heparin; PE, pulmonary embolism; PVC, placental vascular complications; RR, relative risk; SVT, superficial vein thrombosis; UFH, unfractionated heparin; VTE, venous thromboembolism. 
(45.5\%) received aspirin in the BEFORE and AFTER period, respectively. In the AFTER period, compliance with the aspirin treatment suggested by the risk score was $97.4 \%$ overall. A similar compliance with the suggested aspirin treatment was observed for patients presenting a PVC.

\section{Other Data}

Median birth weight was 3,070 and 3,160 g in the BEFORE and AFTER periods of the study, respectively. The Appearance, Pulse, Grimace, Activity, and Respiration score and other parameters did not differ significantly between the two periods (data not shown). No treatment-related adverse events were observed in neonates.

\section{Discussion}

The results of this large multi-centre, controlled BEFORE and AFTER intervention study show that implementation of the STRATHEGE risk scoring system and the proposed prophylactic strategies significantly reduced the risk of VTE and PVC by 50 and $30 \%$, respectively, in at-risk pregnant women. It particularly reduced the rate of severe PVC. Overall, use of our scoring system 16 times may avoid one event. However, the study design does not allow resolution of this controversial issue.

Various risk stratification assessments and scoring systems have been proposed. ${ }^{7,9,12,13}$ However, to the best of our knowledge, this is the first prospective validation of a risk scoring system associated with a proposal of prophylactic strategies. Our scoring system was established, using the DELPHI method, by 19 French experts actively involved in both clinical management and research on VTE and PVC. ${ }^{6}$ This score provides an individual estimation of the risk of thrombosis during pregnancy and puerperium and suggests a corresponding prophylactic strategy. The scoring system was developed before the eighth and ninth ACCP guidelines ${ }^{1,5}$ and is no longer in total agreement with more recent guidelines and publications. For example, we chose to associate VTE and PVC when developing our risk score, although the development of a common prophylactic strategy for these two outcomes is still a matter of debate. ${ }^{13-15}$ However, use of our scoring system and its corresponding prophylactic strategies seemed to similarly decrease rates of both VTE and PVC, thereby supporting our choice. Other discrepancies with past ACCP guidelines (such as a family history of VTE) do not concern the major recommendations, supported by a high level of evidence.

In the current ACCP guidelines, monotherapy with aspirin is recommended for women with inherited thrombophilia and a history of pregnancy complications, except for those with anti-phospholipid antibody syndrome. ${ }^{1}$ According to our scoring system, some of these patients could receive LMWH on top of aspirin. At the time when the scoring system was developed, this strategy was supported by the results of several studies ${ }^{16-18}$ suggesting a benefit of LMWH added to aspirin in the prevention of PVC. However, Rodger et al recently failed to demonstrate a benefit of such an association on the rate of pregnancy complications. ${ }^{19}$ The benefits of such a prophylactic association are probably minor and are still widely debated, and further trials are on-going. ${ }^{13,15,17,20}$
It is worth noting that only $2.7 \%$ of our patients received this kind of association based on the risk scoring system used.

LMWH prophylaxis initiated at the start of the third trimester is also controversial, although VTE can occur in women at any time during pregnancy and puerperium. ${ }^{21}$ In contrast to the ACCP guidelines, ${ }^{1}$ French guidelines recommended initiation of LMWH at the start of the third trimester. $^{2}$ Other French investigators suffered from the same limitation in trial construction. ${ }^{7}$ However, it is worth noting that this timing of the start of prophylaxis applied to less than $3 \%$ of our patients.

Some of our patients were possibly under-treated, although $59(2.8 \%)$ women with a history of thrombosis included in the BEFORE or AFTER phase belonged to this sub-group and none relapsed. The 37 VTE events recorded during pregnancy affected mainly the patients included in the BEFORE phase (27 vs. $10, p<0.002$ ), but only $30 \%$ of women received prophylaxis according to their overall risk score, the others being under-treated. VTE events as a whole were greatly reduced during pregnancy by use of our scoring system to guide prophylaxis $(\mathrm{RR}=0.33[0.16 ; 0.69])$. This reduction was also evident for DVT and PE $(0.37$ [0.15; 0.89], $p=0.02)$ during pregnancy. Reflecting the study design, this reduction was observed mainly during pregnancy rather than puerperium. As patients enrolled in the BEFORE stage were included on the day of delivery, physicians had the opportunity to improve prophylaxis during puerperium. This probably explains the absence of any significant difference in puerperium event rates between the BEFORE and AFTER phases. Similarly, 275 PVC occurred, but PVC rates were significantly reduced by $25 \%$ in women who received prophylaxis according to their overall risk score (comprising $40 \%$ of the total study population). This reduction was particularly evident with respect to preeclampsia $(\mathrm{RR}=0.52[0.36 ; 0.75])$.

Our scoring system has been criticized for its potential to encourage excessive prophylaxis. ${ }^{22}$ Concerning this issue, we emphasize that comparison of the BEFORE and AFTER periods is difficult, because some of the score items were retrospectively collected for the BEFORE phase (the scoring system was voluntarily finalized after this phase so as not to influence practices). However, even in our high-risk population, when the scoring system was applied (AFTER phase), heparin was recommended in only $31.1 \%$ of women during pregnancy and 23.7\% during puerperium.

Regarding safety, no significant treatment-related complications were observed. Post-partum haemorrhage occurred following $3.9 \%$ of deliveries, a result in accordance with the literature. ${ }^{1,23}$ Overall, only $15 \%$ of the women affected had received $\mathrm{LMWH}$ and the incidence of post-partum haemorrhage did not differ significantly between the BEFORE and AFTER phases or between women in whom LMWH prophylaxis had been started during puerperium and those having received LMWH during pregnancy. Furthermore, LMWH prophylaxis was discontinued for delivery and peri-dural analgesia, aspirin being discontinued at 35 weeks. This pattern is concordant with the results of many studies indicating the safety of LMWH during pregnancy/puerperium, with no major bleeds. ${ }^{1,24,25}$ No treatment-related adverse neonatal outcomes were observed. 
Implementation studies (also called quasi-experimental studies) must often be conducted under conditions in which randomized designs are difficult or impossible to put into practice and bias cannot be avoided. Our study was not randomized, and bias may have affected the results. However, the apparent similarity between patients included in the BEFORE and AFTER phases, respectively, suggests minimal selection bias. Maternity unit characteristics were also similar in the two phases. Another potential limitation of this study is that we could not assess the influence of any individual element of our intervention, as this would have necessitated a larger study. Finally, it is important to note that we were able to show the impact of this intervention on both VTE and PVC prevention.

\section{Conclusion}

This prospective before-and-after intervention study in more than 2,000 women at risk of VTE and/or PVC showed that the use of our risk scoring system to guide prophylaxis is safe and effective in reducing the risk of thrombotic events during pregnancy. Use of the STRATHEGE scoring system, developed by experts actively involved in the prevention of VTE and PVC, may be recommended.

\section{What is known about this topic?}

- Venous thromboembolism (VTE) prophylaxis during pregnancy should be individualized.

- No evidence-based easy-to-use tool has been available to guide decisions on VTE prophylaxis for the wide range of pregnant women seen in everyday clinical practice.

\section{What does this paper add?}

- The clinical benefit of the STRATHEGE risk scoreguided prophylaxis remained to be validated. Our before-and-after-intervention study prospectively validated this approach in at-risk women.

- The incidence of adverse vascular events during pregnancy was significantly reduced by $32 \%$.

- Use of the STRATHEGE score to guide thromboprophylaxis may be particularly valuable for non-specialists.

Authors' Contributions

C. Chauleur: Literature search, study design, data collection, data analysis, data interpretation and writing of the manuscript. J. C. Gris: Data interpretation and writing of the manuscript. S. Laporte, C. Chapelle and L. Bertoletti: Data analysis, data interpretation and writing of the manuscript. V. Equy, P. Gaucheran, E. Bazan, O. Dupuis and D. Gallot: Contributed equally to data collection. P. Mismetti: Study design.

STRATHEGE Investigators and STRATHEGE Group: Contributed equally to study design and data collection.
Members of the STRATHEGE Group

Prof. D. Benhamou: Paris - Kremlin-Bicêtre, Dr. C. Biron-Andreani: Montpellier, Dr. J. Y. Borg: Rouen, Prof. C. Chauleur: Saint-Etienne, Dr. J. Conard: Paris, Dr. E. Demaistre: Dijon, Prof. L. Drouet: Lariboisière, Paris, Dr. P. Edelman: Paris, Prof. J. Emmerich: HEGP, Paris, Prof. P. Gaucherand: Lyon, Prof. J. C. Gris: Nîmes, Prof. Y. Gruel: Tours, Prof. B. Jude: Lille, Dr. J. L. Lorenzini: Dijon, Prof. P. Mismetti: Saint-Etienne, Dr. E. Pasquier: Brest, Prof. G. Pernod: Grenoble, Dr. A. Robert: Saint-Antoine, Paris, Dr. B. Tardy: Saint-Etienne, Dr. N. Trillot: Lille.

Members of the STRATHEGE Investigators

Dr. C. Chauleur, Dr. V. Equy, Dr. P. Gaucherand, Dr. E. Bazan, Dr. O. Dupuis, Dr. D. Gallot, Dr A. Favard, Dr. C. Huissoud, Dr. A. Cornu, Dr. J.F. Nord, Dr. C. Houlle, Dr. F. Ruesch, Dr. E. Magne, Dr. R. Ghawi, Dr. P. Mares, Dr. F. Champion, Dr. A.M. Forest, Dr. P. Boulot, Dr. F. Bousquet, Dr. L. Vulliez, Dr. G. Le Maout.

\section{Ethical Approval}

This protocol was approved by the ethics committee of the University Hospital of Saint Etienne on October 19th, 2006; and by the National Commission on Computerization and Freedom (Commission Nationale de l'Informatique et des Libertés [CNIL]): 908179; CTTIRS: 08.120. The study was registered with Clinicaltrials.gov (registry no. NCT0074521).

\section{Funding}

This study was sponsored by the University Hospital of Saint Etienne and supported by grants from the French Ministry of Health (Hospital Clinical Research Program PHRC IR 0508080) in 2005 and 2010, and a grant from Bourse LIVE.

\section{Conflict of Interest}

Céline Chauleur, Jean-Christophe Gris and Patrick Mismetti have received research grants from Sanofi-Aventis, France, but none of the authors reports a substantial direct or indirect commercial or financial incentive associated with publication of this article.

\section{Acknowledgements}

The authors would like to thank the investigators in each centre: CHU Saint-Étienne, CHU Grenoble, Hospices Civils de Lyon (HFME, Hôpital Croix-Rousse, Hôpital Lyon Sud, Hôtel Dieu), CH Montélimar, Polyclinique and CHU Clermont Ferrand, $\mathrm{CH}$ Vichy, $\mathrm{CH}$ Montbrison, $\mathrm{CH}$ Roanne, $\mathrm{CH}$ Saint-Chamond, CH Firminy, CH Albertville CHU Nîmes, CH Montluçon, CHU Montpellier, Clinique Sète, CH Villefranche-Sur-Saône and CH Saint Foy-Lès-Lyon. We would also like to thank all patients for their active participation. We are grateful to Paula Harry for English revision and editorial assistance, and to Dr. Saskia Middeldorp for critical comments. 


\section{References}

1 Bates SM, Greer IA, Middeldorp S, Veenstra DL, Prabulos AM, Vandvik PO. VTE, thrombophilia, antithrombotic therapy, and pregnancy: Antithrombotic Therapy and Prevention of Thrombosis, 9th ed: American College of Chest Physicians EvidenceBased Clinical Practice Guidelines. Chest 2012;141(2, Suppl): e691S-e736S

2 Samama CM, Albaladejo P, Benhamou D, et al; Committee for Good Practice Standards of the French Society for Anaesthesiology and Intensive Care (SFAR). Venous thromboembolism prevention in surgery and obstetrics: clinical practice guidelines. Eur J Anaesthesiol 2006;23(02):95-116

3 Thromboembolic Disease in Pregnancy and the Puerperium. Acute Management. Available at: https://www.rcog.org.uk/globalassets/documents/guidelines/gtg -37b.pdf. Accessed March 5, 2016

4 Duhl AJ, Paidas MJ, Ural SH, et al; Pregnancy and Thrombosis Working Group. Antithrombotic therapy and pregnancy: consensus report and recommendations for prevention and treatment of venous thromboembolism and adverse pregnancy outcomes. Am J Obstet Gynecol 2007;197(05):457.e1-457.e21

5 Bates SM, Greer IA, Pabinger I, et al. American College of Chest Physicians. Venous thromboembolism, thrombophilia, antithrombotic therapy, and pregnancy: American College of Chest Physicians Evidence-Based Clinical Practice Guidelines (8th ed.). Chest 2008;133:844S-886S

6 Chauleur C, Gris JC, Laporte S, et al; STRATHEGE Group. Use of the Delphi method to facilitate antithrombotics prescription during pregnancy. Thromb Res 2010;126(02):88-92

7 Dargaud Y, Rugeri L, Vergnes MC, et al. A risk score for the management of pregnant women with increased risk of venous thromboembolism: a multicentre prospective study. Br J Haematol 2009;145(06):825-835

8 Lindqvist PG, Kublikas M, Dahlbäck B. Individual risk assessment of thrombosis in pregnancy. Acta Obstet Gynecol Scand 2002;81 (05):412-416

9 Bauersachs RM, Dudenhausen J, Faridi A, et al; EThIG Investigators. Risk stratification and heparin prophylaxis to prevent venous thromboembolism in pregnant women. Thromb Haemost 2007;98(06):1237-1245

10 Weiss N, Bernstein PS. Risk factor scoring for predicting venous thromboembolism in obstetric patients. Am J Obstet Gynecol 2000;182(05):1073-1075

11 Sarig G, Vidergor G, Brenner B. Assessment and management of high-risk pregnancies in women with thrombophilia. Blood Rev 2009;23(04):143-147

12 Chauleur C, Quenet S, Varlet MN, et al. Feasibility of an easy-to-use risk score in the prevention of venous thromboembolism and placental vascular complications in pregnant women: a prospective cohort of 2736 women. Thromb Res 2008;122(04):478-484
13 Rodger MA, Carrier M, Le Gal G, et al; Low-Molecular-Weight Heparin for Placenta-Mediated Pregnancy Complications Study Group. Meta-analysis of low-molecular-weight heparin to prevent recurrent placenta-mediated pregnancy complications. Blood 2014;123(06):822-828

14 Simcox LE, Ormesher L, Tower C, Greer IA. Thrombophilia and pregnancy complications. Int J Mol Sci 2015;16(12):28418-28428

15 Areia AL, Fonseca E, Areia M, Moura P. Low-molecular-weight heparin plus aspirin versus aspirin alone in pregnant women with hereditary thrombophilia to improve live birth rate: meta-analysis of randomized controlled trials. Arch Gynecol Obstet 2016; 293(01):81-86

16 Gris JC, Chauleur C, Faillie JL, et al. Enoxaparin for the secondary prevention of placental vascular complications in women with abruptio placentae. The pilot randomised controlled NOH-AP trial. Thromb Haemost 2010;104(04):771-779

17 de Jong PG, Kaandorp S, Di Nisio M, Goddijn M, Middeldorp S. Aspirin and/or heparin for women with unexplained recurrent miscarriage with or without inherited thrombophilia. Cochrane Database Syst Rev 2014;7(07):CD004734

18 Gris JC, Mercier E, Quéré I, et al. Low-molecular-weight heparin versus low-dose aspirin in women with one fetal loss and a constitutional thrombophilic disorder. Blood 2004;103(10): 3695-3699

19 Rodger MA, Hague WM, Kingdom J, et al; TIPPS Investigators. Antepartum dalteparin versus no antepartum dalteparin for the prevention of pregnancy complications in pregnant women with thrombophilia (TIPPS): a multinational open-label randomised trial. Lancet 2014;384(9955):1673-1683

20 de Jong PG, Quenby S, Bloemenkamp KW, et al. ALIFE2 study: lowmolecular-weight heparin for women with recurrent miscarriage and inherited thrombophilia-study protocol for a randomized controlled trial. Trials 2015;16:208

21 Jacobsen AF, Skjeldestad FE, Sandset PM. Ante- and postnatal risk factors of venous thrombosis: a hospital-based case-control study. J Thromb Haemost 2008;6(06):905-912

22 Dargaud Y, Rugeri L, Négrier C, Trzeciak MC, Ninet J. Feasibility of an easy-to-use risk score in the prevention of venous thromboembolism and placental vascular complications in pregnant women. A rebuttal. Thromb Res 2008;122(05):715-716

23 Bateman BT, Berman MF, Riley LE, Leffert LR. The epidemiology of postpartum hemorrhage in a large, nationwide sample of deliveries. Anesth Analg 2010;110(05):1368-1373

24 Freedman RA, Bauer KA, Neuberg DS, Zwicker JI. Timing of postpartum enoxaparin administration and severe postpartum hemorrhage. Blood Coagul Fibrinolysis 2008;19(01): 55-59

25 James A; Committee on Practice Bulletins-Obstetrics. Practice bulletin no. 123: thromboembolism in pregnancy. Obstet Gynecol 2011;118(03):718-729 